\title{
Stability and Separation in Volume Comparison Problems
}

\author{
A. Koldobsky * \\ Department of Mathematics, University of Missouri Columbia, MO 65211
}

\begin{abstract}
We review recent stability and separation results in volume comparison problems and use them to prove several hyperplane inequalities for intersection and projection bodies.
\end{abstract}

Keywords and phrases: convex bodies, sections, projections

Mathematics Subject Classification: 52A20

\section{Introduction}

A typical volume comparison problem asks whether inequalities

$$
f_{K}(\xi) \leq f_{L}(\xi), \quad \forall \xi \in S^{n-1}
$$

imply $|K| \leq|L|$ for any $K, L$ from a certain class of origin-symmetric convex bodies in $\mathbb{R}^{n}$, where $f_{K}$ is a geometric characteristic of $K$. One can have in mind the hyperplane section function $f_{K}(\xi)=\left|K \cap \xi^{\perp}\right|$, where $|K|$ stands for volume of proper dimension and $\xi^{\perp}$ is the central hyperplane perpendicular to $\xi \in S^{n-1}$.

In the case where the answer to a volume comparison problem is affirmative, one can ask a stronger stability question. Suppose that $\varepsilon>0$ and

$$
f_{K}(\xi) \leq f_{L}(\xi)+\varepsilon, \quad \forall \xi \in S^{n-1} .
$$

Does there exist a constant $c$ not dependent on $\varepsilon$ and such that for every $\varepsilon>0$

$$
|K|^{\frac{n-1}{n}} \leq|L|^{\frac{n-1}{n}}+c \varepsilon ?
$$

Stability results are related to hyperplane inequalities as follows. Suppose stability holds for both pairs $K, L$ and $L, K$ with the same constant $c$. Put

$$
\varepsilon=\max _{\xi \in S^{n-1}}\left|f_{K}(\xi)-f_{L}(\xi)\right|,
$$

then one can switch $K$ and $L$ in (1.1) and, correspondingly, in (1.2). The resulting inequality for volumes will be called a volume difference inequality:

$$
\left.|| K\right|^{\frac{n-1}{n}}-|L|^{\frac{n-1}{n}}\left|\leq c \varepsilon=c \max _{\xi \in S^{n-1}}\right| f_{K}(\xi)-f_{L}(\xi) \mid .
$$

\footnotetext{
*Corresponding author. E-mail: koldobskiya@missouri.edu
} 
Suppose now that the function $f_{L}$ converges to zero uniformly with respect to $\xi$ when $L=\beta B_{2}^{n}$ is a multiple of the unit Euclidean ball and $\beta \rightarrow 0$. Then, when $L=\beta B_{2}^{n}$ and $\beta \rightarrow 0$, the inequality (1.3) turns into what we call a hyperplane inequality:

$$
|K|^{\frac{n-1}{n}} \leq c \max _{\xi \in S^{n-1}} f_{K}(\xi)
$$

One can also consider a separation problem. Suppose that $\varepsilon>0$ and

$$
f_{K}(\xi) \leq f_{L}(\xi)-\varepsilon, \quad \forall \xi \in S^{n-1} .
$$

Does there exist a constant $c$ not dependent on $\varepsilon$ and such that for every $\varepsilon>0$

$$
|K|^{\frac{n-1}{n}} \leq|L|^{\frac{n-1}{n}}-c \varepsilon ?
$$

In the case where the answer is affirmative, assuming that

$$
\varepsilon=\min _{\xi \in S^{n-1}}\left(f_{L}(\xi)-f_{K}(\xi)\right)>0
$$

we get another kind of a volume difference inequality:

$$
|L|^{\frac{n-1}{n}}-|K|^{\frac{n-1}{n}} \geq c \varepsilon=c \min _{\xi \in S^{n-1}}\left(f_{L}(\xi)-f_{K}(\xi)\right) .
$$

Again, if $f_{\beta B_{2}^{n}}$ converges to zero uniformly in $\xi$ when $\beta \rightarrow 0$, we get the following version of a hyperplane inequality:

$$
|L|^{\frac{n-1}{n}} \geq c \min _{\xi \in S^{n-1}} f_{L}(\xi) .
$$

This strategy was first applied in [24] to several functions $f_{K}$ including the hyperplane section function and the hyperplane projection function. In [26] similar inequalities were proved for arbitrary measure with continuous density in place of volume. Sections of lower dimensions were considered in [27], and stability and hyperplane inequalities for complex convex bodies were proved in $[25,28]$.

In this article we review stability and separation results and prove some of them with the best possible constants, while in the original papers the constants were sometimes estimated. The proofs are based on recently developed Fourier analytic approach to sections and projections of convex bodies; see [22,30,32]. We also prove several hyperplane inequalities for intersection and projection bodies.

\section{Hyperplane sections}

Suppose that

$$
f_{K}(\xi)=S_{K}(\xi)=\left|K \cap \xi^{\perp}\right|, \quad \xi \in S^{n-1},
$$

is the hyperplane section function, then the volume comparison question is the matter of the BusemannPetty problem, raised in 1956 in [8]. Let $K, L$ be origin-symmetric convex bodies in $\mathbb{R}^{n}$ such that $\left|K \cap \xi^{\perp}\right| \leq\left|L \cap \xi^{\perp}\right|$ for every $\xi \in S^{n-1}$. Does it necessarily follow that $|K| \leq|L|$ ? The problem was solved at the end of the 1990's as the result of a sequence of papers [33], [1], [15], [7], [34], [36], [9], [10], [42], [43], [20], [21], [44], [12] ; see [22, p. 3] or [11, p. 343] for the history of the solution. The answer is affirmative if $n \leq 4$, and it is negative if $n \geq 5$. Moreover, Lutwak [34] proved that if $K$ is an intersection body (see definition below) and $L$ is any origin-symmetric star body, then the answer to the Busemann-Petty problem is affirmative in every dimension.

The corresponding stability result was proved in [24, Theorem 1]. The theorem is stated in [24] with $c_{n}$ replaced by 1 , though the proof there actually establishes the result with the constant $c_{n}$, which is the best possible. Also, the proof in [24] is geometric, while here we use methods of Fourier analysis. 
Throughout the paper

$$
c_{n}:=\frac{\left|B_{2}^{n}\right|^{\frac{n-1}{n}}}{\left|B_{2}^{n-1}\right|},
$$

where $B_{2}^{n}$ is the unit Euclidean ball. Note that $c_{n} \in\left(\frac{1}{\sqrt{e}}, 1\right)$; see for example [29, Lemma 2.1].

Theorem 2.1. ([24]) Suppose that $\varepsilon>0, K$ and $L$ are origin-symmetric star bodies in $\mathbb{R}^{n}$, and $K$ is an intersection body. If for every $\xi \in S^{n-1}$

$$
\left|K \cap \xi^{\perp}\right| \leq\left|L \cap \xi^{\perp}\right|+\varepsilon
$$

then

$$
|K|^{\frac{n-1}{n}} \leq|L|^{\frac{n-1}{n}}+c_{n} \varepsilon
$$

Recall that the constant $c_{n}<1$. To prove Theorem 2.1 we need several definitions and known facts. We say that a closed bounded set $K$ in $\mathbb{R}^{n}$ is a star body if every straight line passing through the origin crosses the boundary of $K$ at exactly two points different from the origin, the origin is an interior point of $K$, and the Minkowski functional of $K$ defined by

$$
\|x\|_{K}=\min \{a \geq 0: x \in a K\}
$$

is a continuous function on $\mathbb{R}^{n}$.

The radial function of a star body $K$ is defined by

$$
\rho_{K}(x)=\|x\|_{K}^{-1}, \quad x \in \mathbb{R}^{n} .
$$

If $x \in S^{n-1}$ then $\rho_{K}(x)$ is the radius of $K$ in the direction of $x$.

Writing volume in polar coordinates we get the polar formula for volume

$$
|K|=\frac{1}{n} \int_{S^{n-1}} \rho_{K}^{n}(\theta) d \theta=\frac{1}{n} \int_{S^{n-1}}\|\theta\|_{K}^{-n} d \theta,
$$

and the polar formula for the volume of a section

$$
\left|K \cap \xi^{\perp}\right|=\frac{1}{n-1} \int_{S^{n-1} \cap \xi^{\perp}} \rho_{K}^{n-1}(\theta) d \theta .
$$

The class of intersection bodies was introduced by Lutwak [34]. Let $K, L$ be origin-symmetric star bodies in $\mathbb{R}^{n}$. We say that $K$ is the intersection body of $L$ if the radius of $K$ in every direction is equal to the $(n-1)$-dimensional volume of the section of $L$ by the central hyperplane orthogonal to this direction, i.e. for every $\xi \in S^{n-1}$,

$$
\rho_{K}(\xi)=\|\xi\|_{K}^{-1}=\left|L \cap \xi^{\perp}\right| .
$$

A more general class of intersection bodies can be defined (see [16]) as the closure of the class of intersection bodes of star bodies in the radial metric

$$
\rho(K, L)=\max _{\xi \in S^{n-1}}\left|\rho_{K}(\xi)-\rho_{L}(\xi)\right| .
$$

We consider Schwartz distributions, i.e. continuous functionals on the space $\mathcal{S}\left(\mathbb{R}^{n}\right)$ of rapidly decreasing infinitely differentiable functions on $\mathbb{R}^{n}$. The Fourier transform of a distribution $f$ is defined by $\langle\hat{f}, \phi\rangle=$ $\langle f, \hat{\phi}\rangle$ for every test function $\phi \in \mathcal{S}\left(\mathbb{R}^{n}\right)$. For any even distribution $f$, we have $(\hat{f})^{\wedge}=(2 \pi)^{n} f$.

If $K$ is a star body and $0<p<n$, then $\|\cdot\|_{K}^{-p}$ is a locally integrable function on $\mathbb{R}^{n}$ and represents a distribution acting by integration. Suppose that $K$ is infinitely smooth, i.e. $\|\cdot\|_{K} \in C^{\infty}\left(S^{n-1}\right)$ is an infinitely differentiable function on the sphere. Then by [22, Lemma 3.16], the Fourier transform of 
$\|\cdot\|_{K}^{-p}$ is an extension of some function $g \in C^{\infty}\left(S^{n-1}\right)$ to a homogeneous function of degree $-n+p$ on $\mathbb{R}^{n}$. When we write $\left(\|\cdot\|_{K}^{-p}\right)^{\wedge}(\xi)$, we mean $g(\xi), \xi \in S^{n-1}$. If $K, L$ are infinitely smooth star bodies, the following spherical version of Parseval's formula was proved in [23] (see [22, Lemma 3.22]): for any $p \in(-n, 0)$

$$
\int_{S^{n-1}}\left(\|\cdot\|_{K}^{-p}\right)^{\wedge}(\xi)\left(\|\cdot\|_{L}^{-n+p}\right)^{\wedge}(\xi)=(2 \pi)^{n} \int_{S^{n-1}}\|x\|_{K}^{-p}\|x\|_{L}^{-n+p} d x .
$$

A distribution is called positive definite if its Fourier transform is a positive distribution in the sense that $\langle\hat{f}, \phi\rangle \geq 0$ for every non-negative test function $\phi$. It was proved in [20, Theorem 1] that an originsymmetric star body in $\mathbb{R}^{n}$ is an intersection body if and only if the function $\|\cdot\|_{K}^{-1}$ represents a positive definite distribution. As proved in $[10,44]$ (see also [12] or [22, p. 73]), every origin-symmetric convex body in $\mathbb{R}^{n}, n \leq 4$ is an intersection body. It was shown in [20, Theorem 3] that the unit ball of any finite dimensional subspace of $L_{p}, 0<p \leq 2$ is an intersection body. For other results on intersection bodies, see [11, Chapter 8] and [22, Chapter 4].

For origin-symmetric star bodies $K, L$ in $\mathbb{R}^{n}$, the radial sum $K+{ }_{r} L$ of $K$ and $L$ is a star body defined by

$$
\rho_{K+{ }_{r} L}(\xi)=\rho_{K}(\xi)+\rho_{L}(\xi), \quad \forall \xi \in S^{n-1} .
$$

If $K$ and $L$ are both intersection bodies, then their radial sum is also an intersection body, which follows, for example, from the Fourier characterization of intersection bodies formulated above.

Proof of Theorem 2.1. By approximation (see, for example [39, Theorem 3.3.1]), we can assume that the bodies $K$ and $L$ are infinitely smooth. It was proved in [19] that

$$
\left|K \cap \xi^{\perp}\right|=\frac{1}{\pi(n-1)}\left(\|x\|_{K}^{-n+1}\right)^{\wedge}(\xi), \quad \forall \xi \in S^{n-1},
$$

so (2.1) can be written as

$$
\left(\|x\|_{K}^{-n+1}\right)^{\wedge}(\xi) \leq\left(\|x\|_{L}^{-n+1}\right)^{\wedge}(\xi)+\pi(n-1) \varepsilon, \quad \forall \xi \in S^{n-1} .
$$

Also, by the remark before the proof and the Fourier characterization of intersection bodies, $\left(\|x\|_{K}^{-1}\right)^{\wedge}$ is an infinitely smooth non-negative function on the sphere. By (2.7), the polar formula for volume $(2.2)$ and Parseval's formula on the sphere (2.5),

$$
\begin{gathered}
(2 \pi)^{n} n|K|=(2 \pi)^{n} \int_{S^{n-1}}\|x\|_{K}^{-n+1}\|x\|_{K}^{-1} d x \\
=\int_{S^{n-1}}\left(\|x\|_{K}^{-1}\right)^{\wedge}(\theta)\left(\|x\|_{K}^{-n+1}\right)^{\wedge}(\theta) d \theta \\
\leq \int_{S^{n-1}}\left(\|x\|_{K}^{-1}\right)^{\wedge}(\theta)\left(\|x\|_{L}^{-n+1}\right)^{\wedge}(\theta) d \theta \\
\quad+\pi(n-1) \varepsilon \int_{S^{n-1}}\left(\|x\|_{K}^{-1}\right)^{\wedge}(\theta) d \theta .
\end{gathered}
$$

By Parseval's formula and Hölder's inequality,

$$
\begin{gathered}
\int_{S^{n-1}}\left(\|x\|_{K}^{-1}\right)^{\wedge}(\theta)\left(\|x\|_{L}^{-n+1}\right)^{\wedge}(\theta) d \theta \\
=(2 \pi)^{n} \int_{S^{n-1}}\|x\|_{L}^{-n+1}\|x\|_{K}^{-1} d x \leq(2 \pi)^{n} n|K|^{\frac{1}{n}}|L|^{\frac{n-1}{n}} .
\end{gathered}
$$

To estimate the second summand in (2.8), we use the formula for the Fourier transform (in the sense of distributions; see [13, p.194])

$$
\left(|x|_{2}^{-n+1}\right)^{\wedge}(\theta)=\frac{2 \pi^{\frac{n+1}{2}}}{\Gamma\left(\frac{n-1}{2}\right)}|\theta|_{2}^{-1} .
$$


Again using Parseval's formula and then Hölder's inequality,

$$
\begin{gathered}
\int_{S^{n-1}}\left(\|x\|_{K}^{-1}\right)^{\wedge}(\theta) d \theta \\
=\frac{\Gamma\left(\frac{n-1}{2}\right)}{2 \pi^{\frac{n+1}{2}}} \int_{S^{n-1}}\left(\|x\|_{K}^{-1}\right)^{\wedge}(\theta)\left(|x|_{2}^{-n+1}\right)^{\wedge}(\theta) d \theta \\
=\frac{(2 \pi)^{n} \Gamma\left(\frac{n-1}{2}\right)}{2 \pi^{\frac{n+1}{2}}} \int_{S^{n-1}}\|x\|_{K}^{-1} d x \\
\leq \frac{(2 \pi)^{n} \Gamma\left(\frac{n-1}{2}\right)\left|S^{n-1}\right|^{\frac{n-1}{n}}}{2 \pi^{\frac{n+1}{2}}}\left(\int_{S^{n-1}}\|x\|_{K}^{-n} d x\right)^{\frac{1}{n}} \\
=\frac{(2 \pi)^{n} \Gamma\left(\frac{n-1}{2}\right)\left|S^{n-1}\right|^{\frac{n-1}{n}}}{2 \pi^{\frac{n+1}{2}}}(n|K|)^{\frac{1}{n}}
\end{gathered}
$$

Combining this with $(2.8)$ and (2.9), we get

$$
\begin{gathered}
(2 \pi)^{n} n|K| \leq(2 \pi)^{n} n|K|^{\frac{1}{n}}|L|^{\frac{n-1}{n}} \\
+\frac{(2 \pi)^{n} \varepsilon \pi(n-1) n^{\frac{1}{n}} \Gamma\left(\frac{n-1}{2}\right)\left|S^{n-1}\right|^{\frac{n-1}{n}}}{2 \pi^{\frac{n+1}{2}}}|K|^{1 / n} .
\end{gathered}
$$

Now to represent the coefficient in the required form use

$$
\left|S^{n-1}\right|=n\left|B_{2}^{n}\right|=\frac{2 \pi^{\frac{n}{2}}}{\Gamma\left(\frac{n}{2}\right)} .
$$

Interchanging $K$ and $L$ in Theorem 2.1, we get the corresponding volume difference inequality.

Corollary 2.2. If $K$ and $L$ are intersection bodies in $\mathbb{R}^{n}$ (in particular, any origin-symmetric convex bodies in $\mathbb{R}^{3}$ or $\left.\mathbb{R}^{4}\right)$, then

$$
\left.|| K\right|^{\frac{n-1}{n}}-|L|^{\frac{n-1}{n}}\left|\leq c_{n} \max _{\xi \in S^{n-1}}\right|\left|K \cap \xi^{\perp}\right|-\left|L \cap \xi^{\perp}\right| \mid
$$

If $L=\delta B_{2}^{n}$ in the latter inequality, then sending $\delta$ to zero we get that for any intersection body $K$ in $\mathbb{R}^{n}$ and, in particular, any origin-symmetric convex body in $\mathbb{R}^{3}$ or $\mathbb{R}^{4}$,

$$
|K|^{\frac{n-1}{n}} \leq c_{n} \max _{\xi \in S^{n-1}}\left|K \cap \xi^{\perp}\right| .
$$

Inequality (2.10) also immediately follows from the affirmative part of the Busemann-Petty problem; see [11, Theorem 9.4.11]. Note that (2.10) is a particular case of the well-known and still open Hyperplane Problem (see $[3-5,35]$ ) which can be formulated as follows. Does there exist an absolute constant $C$ so that for any origin-symmetric convex body $K$ in $\mathbb{R}^{n}$

$$
|K|^{\frac{n-1}{n}} \leq C \max _{\xi \in S^{n-1}}\left|K \cap \xi^{\perp}\right|
$$

The best-to-date estimate $C \sim n^{1 / 4}$ belongs to Klartag [18], who slightly improved the previous estimate of Bourgain [6]. 
The volume difference inequality of Corollary 2.2 can also be used to prove a hyperplane inequality for the average volume of central hyperplane sections, which we denote by

$$
\operatorname{as}(K)=\frac{1}{\left|S^{n-1}\right|} \int_{S^{n-1}}\left|K \cap \xi^{\perp}\right| d \xi
$$

For any continuous function $h$ on $S^{n-1}$,

$$
\left|S^{n-2}\right| \int_{S^{n-1}} h(x) d x=\int_{S^{n-1}}\left(\int_{S^{n-1} \cap \xi^{\perp}} h(x) d x\right) d \xi .
$$

Using this and (2.3),

$$
\begin{gathered}
\operatorname{as}(K)=\frac{1}{(n-1)\left|S^{n-1}\right|} \int_{S^{n-1}}\left(\int_{S^{n-1} \cap \xi^{\perp}} \rho_{K}^{n-1}(\theta) d \theta\right) d \xi \\
=\frac{\left|S^{n-2}\right|}{(n-1)\left|S^{n-1}\right|} \int_{S^{n-1}} \rho_{K}^{n-1}(\theta) d \theta .
\end{gathered}
$$

Corollary 2.3. If $K$ is an intersection body in $\mathbb{R}^{n}, n \geq 3$, then

$$
\operatorname{as}(K) \leq \frac{\left|B_{2}^{n-1}\right|}{\left|B_{2}^{n-2}\right|\left|B_{2}^{n}\right|^{\frac{1}{n}}} \max _{\xi \in S^{n-1}} \operatorname{as}\left(K \cap \xi^{\perp}\right)|K|^{\frac{1}{n}},
$$

with equality when $K=B_{2}^{n}$.

Proof : Since $K$ and $B_{2}^{n}$ are intersection bodies, for every $\varepsilon>0$ the radial sum $K{ }_{r} \varepsilon B_{2}^{n}$ is also an intersection body. By Corollary 2.2 applied to the bodies $K{ }_{r_{r}} \varepsilon B_{2}^{n}$ and $K$, we get that for every $\varepsilon>0$

$$
\frac{\left|K+_{r} \varepsilon B_{2}^{n}\right|^{\frac{n-1}{n}}-|K|^{\frac{n-1}{n}}}{\varepsilon} \leq c_{n} \max _{\xi \in S^{n-1}} \frac{\left|\left(K \cap \xi^{\perp}\right)+{ }_{r} \varepsilon B_{2}^{n-1}\right|-\left|K \cap \xi^{\perp}\right|}{\varepsilon} .
$$

By the polar formula for the volume (2.2),

$$
\left|K{ }_{r} \varepsilon B_{2}^{n}\right|=\frac{1}{n} \int_{S^{n-1}}\left(\rho_{K}(\theta)+\varepsilon\right)^{n} d \theta
$$

so

$$
\begin{gathered}
\lim _{\varepsilon \rightarrow 0} \frac{\left|K+_{r} \varepsilon B_{2}^{n}\right|^{\frac{n-1}{n}}-|K|^{\frac{n-1}{n}}}{\varepsilon}=\frac{n-1}{n}|K|^{-\frac{1}{n}} \int_{S^{n-1}} \rho_{K}^{n-1}(\theta) \\
=\frac{n-1}{n}|K|^{-\frac{1}{n}} \frac{(n-1)\left|S^{n-1}\right|}{\left|S^{n-2}\right|} \operatorname{as}(K) .
\end{gathered}
$$

Similarly, the limit of the right-hand side of (2.12), as $\varepsilon \rightarrow 0$, is equal to

$$
c_{n} \frac{(n-2)\left|S^{n-2}\right|}{\left|S^{n-3}\right|} \max _{\xi \in S^{n-1}} \operatorname{as}\left(K \cap \xi^{\perp}\right) .
$$

It is easily seen that the convergence of the quotient in the right-hand side of (2.12) is uniform with respect to $\xi$, as $\varepsilon \rightarrow 0$, so one can switch the limit and maximum. Sending $\varepsilon$ to 0 in (2.12) and using $\left|S^{n-1}\right|=n\left|B_{2}^{n}\right|$ we get the result. 
A separation result for hyperplane sections was proved in [24, Theorem 2]. The constant $c$ in this result does not depend on $\varepsilon$, but depends on the dimension and on the normalized inradius of $K$ :

$$
r(K)=\frac{\min _{\xi \in S^{n-1}} \rho_{K}(\xi)}{|K|^{1 / n}} .
$$

Theorem 2.4. ([24]) Let $K$ and $L$ be origin-symmetric star bodies in $\mathbb{R}^{n}$ and $\varepsilon>0$. Assume that $K$ is an intersection body. If for every $\xi \in S^{n-1}$

$$
\left|K \cap \xi^{\perp}\right| \leq\left|L \cap \xi^{\perp}\right|-\varepsilon
$$

then

$$
|K|^{\frac{n-1}{n}} \leq|L|^{\frac{n-1}{n}}-\sqrt{\frac{2 \pi}{n+1}} r(K) \varepsilon
$$

Since the answer to the Busemann-Petty problem is negative in most dimensions, one may ask what information about the hyperplane section function $S_{K}$ does allow to compare the volumes in all dimensions. An answer to this question was given in [31]: for two origin-symmetric infinitely smooth bodies $K, L$ in $\mathbb{R}^{n}$ and $\alpha \in[n-4, n-1)$ the inequalities

$$
(-\Delta)^{\alpha / 2} S_{K}(\xi) \leq(-\Delta)^{\alpha / 2} S_{L}(\xi), \quad \forall \xi \in S^{n-1}
$$

imply that $|K| \leq|L|$, while for $\alpha<n-4$ this is not necessarily true. Here $\Delta$ is the Laplace operator on $\mathbb{R}^{n}$, and the fractional powers of the Laplacian are defined by

$$
(-\Delta)^{\alpha / 2} f=\frac{1}{(2 \pi)^{n}}\left(|x|_{2}^{\alpha} \hat{f}(x)\right)^{\wedge}
$$

where the Fourier transform is considered in the sense of distributions, $|x|_{2}$ stands for the Euclidean norm in $\mathbb{R}^{n}$, and the function $S_{K}$ is extended in (2.14) to a homogeneous function of degree -1 on the whole $\mathbb{R}^{n}$. The corresponding stability result was proved in [24, Theorem 3$]$.

Theorem 2.5. ([24]) Let $\varepsilon>0, \alpha \in[n-4, n-1$ ), and let $K$ and $L$ be origin-symmetric infinitely smooth convex bodies in $\mathbb{R}^{n}, n \geq 4$, so that for every $\xi \in S^{n-1}$

$$
(-\Delta)^{\alpha / 2} S_{K}(\xi) \leq(-\Delta)^{\alpha / 2} S_{L}(\xi)+\varepsilon .
$$

Then

$$
|K|^{\frac{n-1}{n}} \leq|L|^{\frac{n-1}{n}}+c \varepsilon
$$

where

$$
c=c(\alpha, n)=\frac{\sqrt{\pi}(n-1) \Gamma\left(\frac{n-\alpha-1}{2}\right)}{2^{\alpha+\frac{1}{n}} n^{\frac{n-1}{n}} \Gamma\left(\frac{\alpha+1}{2}\right)\left(\Gamma\left(\frac{n}{2}\right)\right)^{\frac{n-1}{n}}} .
$$

A separation result was proved in [24, Theorem 4].

Theorem 2.6. ([24]) Let $\varepsilon>0, \alpha \in[n-4, n-1), K$ and $L$ be origin-symmetric infinitely smooth convex bodies in $\mathbb{R}^{n}, n \geq 4$, so that for every $\xi \in S^{n-1}$

$$
(-\Delta)^{\alpha / 2} S_{K}(\xi) \leq(-\Delta)^{\alpha / 2} S_{L}(\xi)-\varepsilon .
$$

Then

$$
|K|^{\frac{n-1}{n}} \leq|L|^{\frac{n-1}{n}}-c \varepsilon,
$$

where

$$
c=r(K) \frac{\pi(n-1) \Gamma\left(\frac{n-\alpha-1}{2}\right)}{n 2^{\alpha} \Gamma\left(\frac{\alpha+1}{2}\right) \Gamma\left(\frac{n}{2}\right)} .
$$




\section{Hyperplane projections}

Now we pass to the hyperplane projection function

$$
f_{K}(\xi)=P_{K}(\xi)=|K| \xi^{\perp} \mid,
$$

where $K \mid \xi^{\perp}$ is the orthogonal projection of $K$ to the hyperplane $\xi^{\perp}$. The corresponding volume comparison result is known as Shephard's problem, which was posed in 1964 in [40] and solved soon after that by Petty [37] and Schneider [38], independently. Suppose that $K$ and $L$ are origin-symmetric convex bodies in $\mathbb{R}^{n}$ so that $|K| \xi^{\perp}|\leq| L\left|\xi^{\perp}\right|$ for every $\xi \in S^{n-1}$. Does it follow that $|K| \leq|L|$ ? The answer if affirmative only in dimension 2. Both solutions use the fact that the answer to Shephard's problem is affirmative in every dimension under the additional assumption that $L$ is a projection body; see definition below.

In the case of projections the constant in the stability result depends on the body and dimension, while the constant in the separation result does not. One can say that separation is a more natural property for projections than stability, while for sections it is the other way around. Therefore, we start with a separation result which was proved in [24, Theorem 6]. The constant $c_{n}$ in [24] was at the last moment estimated from below by $1 / \sqrt{e}$, so we now formulate and prove the result with the best possible constant.

Theorem 3.1. ([24]) Suppose that $\varepsilon>0, K$ and $L$ are origin-symmetric convex bodies in $\mathbb{R}^{n}$, and $L$ is a projection body. If for every $\xi \in S^{n-1}$

$$
|K| \xi^{\perp}|\leq| L\left|\xi^{\perp}\right|-\varepsilon
$$

then

$$
|K|^{\frac{n-1}{n}} \leq|L|^{\frac{n-1}{n}}-c_{n} \varepsilon .
$$

To prove Theorem 3.1 we need several more definitions and results from convex geometry. We refer the reader to [39] for details.

The support function of a convex body $K$ in $\mathbb{R}^{n}$ is defined by

$$
h_{K}(x)=\max _{\left\{\xi \in \mathbb{R}^{n}:\|\xi\|_{K}=1\right\}}(x, \xi), \quad x \in \mathbb{R}^{n} .
$$

If $K$ is origin-symmetric, then $h_{K}$ is a norm on $\mathbb{R}^{n}$.

The surface area measure $S(K, \cdot)$ of a convex body $K$ in $\mathbb{R}^{n}$ is defined as follows. For every Borel set $E \subset S^{n-1}, S(K, E)$ is equal to Lebesgue measure of the part of the boundary of $K$ where normal vectors belong to $E$. We usually consider bodies with absolutely continuous surface area measures. A convex body $K$ is said to have the curvature function

$$
f_{K}: S^{n-1} \rightarrow \mathbb{R}
$$

if its surface area measure $S(K, \cdot)$ is absolutely continuous with respect to Lebesgue measure $\sigma_{n-1}$ on $S^{n-1}$, and

$$
\frac{d S(K, \cdot)}{d \sigma_{n-1}}=f_{K} \in L_{1}\left(S^{n-1}\right),
$$

so $f_{K}$ is the density of $S(K, \cdot)$.

By the approximation argument of [39, Th. 3.3.1], we may assume in the formulation of Shephard's problem that the bodies $K$ and $L$ are such that their support functions $h_{K}, h_{L}$ are infinitely smooth functions on $\mathbb{R}^{n} \backslash\{0\}$. Using [22, Lemma 3.16] we get in this case that the Fourier transforms $\widehat{h_{K}}, \widehat{h_{L}}$ are the extensions of infinitely differentiable functions on the sphere to homogeneous distributions on $\mathbb{R}^{n}$ of degree $-n-1$. Moreover, by a similar approximation argument (see also [17, Section 5$]$ ), we may assume that our bodies have absolutely continuous surface area measures. Therefore, in the rest of this section, $K$ and $L$ are convex symmetric bodies with infinitely smooth support functions and absolutely continuous surface area measures. 
The following version of Parseval's formula was proved in [30] (see also [22, Lemma 8.8]):

$$
\int_{S^{n-1}} \widehat{h_{K}}(\xi) \widehat{f_{L}}(\xi) d \xi=(2 \pi)^{n} \int_{S^{n-1}} h_{K}(x) f_{L}(x) d x .
$$

The volume of a body can be expressed in terms of its support function and curvature function:

$$
|K|=\frac{1}{n} \int_{S^{n-1}} h_{K}(x) f_{K}(x) d x
$$

If $K$ and $L$ are two convex bodies in $\mathbb{R}^{n}$ the mixed volume $V_{1}(K, L)$ is equal to

$$
V_{1}(K, L)=\frac{1}{n} \lim _{\varepsilon \rightarrow+0} \frac{|K+\epsilon L|-|K|}{\varepsilon} .
$$

We use the following first Minkowski inequality (see [22, p.23]): for any convex bodies $K, L$ in $\mathbb{R}^{n}$,

$$
V_{1}(K, L) \geq|K|^{\frac{n-1}{n}}|L|^{\frac{1}{n}}
$$

The mixed volume can also be expressed in terms of the support and curvature functions:

$$
V_{1}(K, L)=\frac{1}{n} \int_{S^{n-1}} h_{L}(x) f_{K}(x) d x .
$$

Let $K$ be an origin-symmetric convex body in $\mathbb{R}^{n}$. The projection body $\Pi K$ of $K$ is defined as an origin-symmetric convex body in $\mathbb{R}^{n}$ whose support function in every direction is equal to the volume of the hyperplane projection of $K$ to this direction: for every $\theta \in S^{n-1}$,

$$
h_{\Pi K}(\theta)=|K| \theta^{\perp} \mid .
$$

If $L$ is the projection body of some convex body, we simply say that $L$ is a projection body. The Minkowski (vector) sum of projection bodies is also a projection body. Every projection body is the limit in the Hausdorff metric of Minkowski sums of symmetric intervals. An origin-symmetric convex body in $\mathbb{R}^{n}$ is a projection body if and only if the polar body is the unit ball of an $n$-dimensional subspace of $L_{1}$; see $[11,22,39]$ for proofs and more properties of projection bodies.

Proof of Theorem 3.1. By approximation (see [39, Theorem 3.3.1]), we can assume that $K, L$ are infinitely smooth. It was proved in [30] that

$$
P_{K}(\xi)=|K| \xi^{\perp} \mid=-\frac{1}{\pi} \widehat{f_{K}}(\xi), \quad \forall \xi \in S^{n-1},
$$

where $f_{K}$ is extended from the sphere to a homogeneous function of degree $-n-1$ on the whole $\mathbb{R}^{n}$, and the Fourier transform $\widehat{f_{K}}$ is the extension of a continuous function $P_{K}$ on the sphere to a homogeneous of degree 1 function on $\mathbb{R}^{n}$.

Therefore, the condition (3.1) can be written as

$$
\frac{1}{\pi} \widehat{f_{K}}(\xi) \geq \frac{1}{\pi} \widehat{f_{L}}(\xi)+\varepsilon, \quad \forall \xi \in S^{n-1} .
$$

It was also proved in [30] that an infinitely smooth origin-symmetric convex body $L$ in $\mathbb{R}^{n}$ is a projection body if and only if $\widehat{h_{L}} \leq 0$ on the sphere $S^{n-1}$. Therefore, integrating (3.8) with respect to a negative density,

$$
\int_{S^{n-1}} \widehat{h_{L}}(\xi) \widehat{f_{L}}(\xi) d \xi \geq \int_{S^{n-1}} \widehat{h_{L}}(\xi) \widehat{f_{K}}(\xi) d \xi-\pi \varepsilon \int_{S^{n-1}} \widehat{h_{L}}(\xi) d \xi
$$


Using this, (3.3) and (3.2), we get

$$
\begin{gathered}
(2 \pi)^{n} n|L|=(2 \pi)^{n} \int_{S^{n-1}} h_{L}(x) f_{L}(x) d x=\int_{S^{n-1}} \widehat{h_{L}}(\xi) \widehat{f_{L}}(\xi) d \xi \\
\geq \int_{S^{n-1}} \widehat{h_{L}}(\xi) \widehat{f_{K}}(\xi) d \xi-\pi \varepsilon \int_{S^{n-1}} \widehat{h_{L}}(\xi) d \xi \\
=(2 \pi)^{n} \int_{S^{n-1}} h_{L}(x) f_{K}(x) d x-\pi \varepsilon \int_{S^{n-1}} \widehat{h_{L}}(\xi) d \xi .
\end{gathered}
$$

We estimate the first summand from below using the first Minkowski inequality:

$$
(2 \pi)^{n} \int_{S^{n-1}} h_{L}(x) f_{K}(x) d x \geq(2 \pi)^{n} n\left(\operatorname{Vol}_{n}(L)\right)^{\frac{1}{n}}\left(\operatorname{Vol}_{n}(K)\right)^{\frac{n-1}{n}} .
$$

To estimate the second term in (3.9), note that, by (3.7), the Fourier transform of the curvature function of the Euclidean ball

$$
\widehat{f_{2}}(\xi)=-\pi\left|B_{2}^{n-1}\right| .
$$

Therefore, by Parseval's formula, (3.5) and the first Minkowski inequality,

$$
\begin{gathered}
\pi \varepsilon \int_{S^{n-1}} \widehat{h_{L}}(\xi) d \xi=-\frac{\varepsilon}{\left|B_{2}^{n-1}\right|} \int_{S^{n-1}} \widehat{h_{L}}(\xi) \widehat{f_{2}}(\xi) d \xi \\
=-\frac{(2 \pi)^{n} \varepsilon}{\left|B_{2}^{n-1}\right|} \int_{S^{n-1}} h_{L}(x) f_{2}(x) d x=-\frac{(2 \pi)^{n} \varepsilon}{\left|B_{2}^{n-1}\right|} n V_{1}\left(B_{2}^{n}, L\right) \\
\leq-\frac{(2 \pi)^{n} n \varepsilon}{\left|B_{2}^{n-1}\right|}|L|^{\frac{1}{n}}\left|B_{2}^{n}\right|^{\frac{n-1}{n}}=-(2 \pi)^{n} n \varepsilon c_{n}|L|^{\frac{1}{n}} .
\end{gathered}
$$

Combining this with (3.9) and (3.10), we get the result.

As explained in the Introduction, the separation result of Theorem 3.1 leads to a volume difference inequality of the type (1.7).

Corollary 3.2. If $L$ is a projection body in $\mathbb{R}^{n}$ and $K$ is an arbitrary origin-symmeric convex body in $\mathbb{R}^{n}$ so that

$$
\min _{\xi \in S^{n-1}}\left(|L| \xi^{\perp}|-| K\left|\xi^{\perp}\right|\right)>0
$$

then

$$
|L|^{\frac{n-1}{n}}-|K|^{\frac{n-1}{n}} \geq c_{n} \min _{\xi \in S^{n-1}}\left(|L| \xi^{\perp}|-| K\left|\xi^{\perp}\right|\right) .
$$

Putting $K=\beta B_{2}^{n}$ in (3.11) and sending $\beta \rightarrow 0$, we get a hyperplane inequality of the type (1.8), which was earlier deduced directly from the solution to Shephard's problem in [11, Corollary 9.3.4]: if $L$ is a projection body in $\mathbb{R}^{n}$, then

$$
|L|^{\frac{n-1}{n}} \geq c_{n} \min _{\xi \in S^{n-1}}|L| \xi^{\perp} \mid
$$

Recall that $c_{n}>1 / \sqrt{e}$. For general symmetric convex bodies, Ball [2] proved that $c_{n}$ may and has to be replaced in (3.12) by $c / \sqrt{n}$, where $c$ is an absolute constant. Also, note that the inequality

$$
|L|^{\frac{n-1}{n}} \leq c_{n} \max _{\xi \in S^{n-1}}|L| \xi^{\perp} \mid
$$

holds for all origin-symmetric convex bodies and follows from the Cauchy projection formula for the surface area (the first part of (3.15)) and the classical isoperimetric inequality; see [11, p. 363].

The volume difference inequality (3.11) allows to prove a hyperplane inequality for the surface area of projection bodies. 
Corollary 3.3. Let $L$ be a projection body in $\mathbb{R}^{n}, n \geq 3$, then the surface area

$$
S(L) \geq \frac{n}{n-1} c_{n} \min _{\xi \in S^{n-1}} S\left(L \mid \xi^{\perp}\right)|L|^{\frac{1}{n}} .
$$

Proof : The surface area of $L$ can be computed as

$$
S(L)=\lim _{\varepsilon \rightarrow+0} \frac{\left|L+\varepsilon B_{2}^{n}\right|-|L|}{\varepsilon} .
$$

For every $\varepsilon>0$ the Minkowski sum $L+\varepsilon B_{2}^{n}$ is also a projection body. The inequality (3.11) with the bodies $L+\varepsilon B_{2}^{n}$ and $L$ in place of $L$ and $K$ implies

$$
\frac{\left|L+\varepsilon B_{2}^{n}\right|^{\frac{n-1}{n}}-|L|^{\frac{n-1}{n}}}{\varepsilon} \geq c_{n} \min _{\xi \in S^{n-1}} \frac{\left|\left(L \mid \xi^{\perp}\right)+\varepsilon B_{2}^{n-1}\right|-|L| \xi^{\perp} \mid}{\varepsilon} .
$$

By the Minkowski theorem on mixed volumes ([39, Theorem 5.1.6] or [11, Theorem A.3.1]),

$$
\frac{\left|\left(L \mid \xi^{\perp}\right)+\varepsilon B_{2}^{n-1}\right|-|L| \xi^{\perp} \mid}{\varepsilon}=\sum_{i=1}^{n-1}\left(\begin{array}{c}
n-1 \\
i
\end{array}\right) W_{i}\left(L \mid \xi^{\perp}\right) \varepsilon^{i-1},
$$

where $W_{i}$ are quermassintegrals. The function $\xi \mapsto L \mid \xi^{\perp}$ is continuous from $S^{n-1}$ to the class of originsymmetric convex sets equiped with the Hausdorff metric, and $W_{i}$ 's are also continuous with respect to this metric (see [39, p. 275]), so the functions $\xi \mapsto W_{i}\left(L \mid \xi^{\perp}\right)$ are continuous and, hence, bounded on the sphere. This implies that the left-hand side of (3.14) converges to $S\left(L \mid \xi^{\perp}\right)$, as $\varepsilon \rightarrow 0$, uniformly with respect to $\xi$. The latter allows to switch the limit and maximum in the right-hand side of (3.13), as $\varepsilon \rightarrow 0$. Sending $\varepsilon$ to zero in (3.13), we get

$$
\frac{n-1}{n}|L|^{-1 / n} S(L) \geq c_{n} \min _{\xi \in S^{n-1}} S\left(L \mid \xi^{\perp}\right) .
$$

Note the similarity between Corollaries 3.3 and 2.3. In fact, the Cauchy projection formula (see for example [11, p. 408]) can be written as

$$
S(L)=\frac{1}{\left|B_{2}^{n-1}\right|} \int_{S^{n-1}}|L| \xi^{\perp} \mid d \xi=\frac{\left|S^{n-1}\right|}{\left|B_{2}^{n-1}\right|} \operatorname{ap}(L),
$$

where we denote by $\operatorname{ap}(L)$ the average hyperplane projection of $L$. Thus, the inequality of Corollary 3.3 turns into

$$
\operatorname{ap}(L) \geq \frac{\left|B_{2}^{n-1}\right|}{\left|B_{2}^{n-2}\right|\left|B_{2}^{n}\right|^{\frac{1}{n}}} \min _{\xi \in S^{n-1}} \operatorname{ap}\left(L \mid \xi^{\perp}\right)|L|^{\frac{1}{n}} .
$$

A stability result for hyperplane projections was proved in [24, Theorem 5]. Define the normalized circumradius of $L$ by

$$
R(L)=\frac{\max _{\xi \in S^{n-1}} \rho_{L}(\xi)}{|L|^{\frac{1}{n}}} .
$$

Theorem 3.4. ([24]) Suppose that $\varepsilon>0, K$ and $L$ are origin-symmetric convex bodies in $\mathbb{R}^{n}$, and $L$ is a projection body. If for every $\xi \in S^{n-1}$

$$
|K| \xi^{\perp}|\leq| L\left|\xi^{\perp}\right|+\varepsilon
$$

then

$$
|K|^{\frac{n-1}{n}} \leq|L|^{\frac{n-1}{n}}+\sqrt{\frac{2 \pi}{n}} R(L) \varepsilon
$$


Since the answer to Shephard's problem is negative in most dimensions, one can ask what condition on the hyperplane projection function does imply the inequality for volumes. Yaskin [41] proved that for $\alpha \in[n, n+1)$ the inequalities

$$
(-\Delta)^{\alpha / 2} P_{K}(\xi) \geq(-\Delta)^{\alpha / 2} P_{L}(\xi), \quad \forall \xi \in S^{n-1}
$$

imply that $|K| \leq|L|$, where the projection functions are extended to homogeneous functions of degree 1 on the whole $\mathbb{R}^{n}$. The latter result is no longer true for $\alpha<n$. We end this section by formulating the stability version of the result of Yaskin.

Theorem 3.5. Let $\varepsilon>0, \alpha \in[n, n+1), K$ and $L$ be origin-symmetric infinitely smooth convex bodies in $\mathbb{R}^{n}, n \geq 3$, so that for every $\xi \in S^{n-1}$

$$
(-\Delta)^{\alpha / 2} P_{K}(\xi) \leq(-\Delta)^{\alpha / 2} P_{L}(\xi)+\varepsilon
$$

Then

$$
|K|^{\frac{n-1}{n}} \leq|L|^{\frac{n-1}{n}}+c \varepsilon
$$

where

$$
c=\frac{\Gamma\left(\frac{n-\alpha+1}{2}\right)\left|S^{n-1}\right| R(L)}{2^{\alpha+1} \pi^{\frac{n}{2}} \Gamma\left(\frac{\alpha+1}{2}\right) n} .
$$

Note that this is no longer true if $\alpha<n$, because the underlying comparison result fails, as shown in [41].

\section{Arbitrary measures}

Zvavitch [46] found a remarkable generalization of the Busemann-Petty problem to arbitrary measures, namely, one can replace volume by any measure with even continuous density in $\mathbb{R}^{n}$. In particular, if $K$ is an intersection body in $\mathbb{R}^{n}$ and $L$ is an arbitrary origin-symmetric star body in $\mathbb{R}^{n}$, then the inequalities

$$
\mu\left(K \cap \xi^{\perp}\right) \leq \mu\left(L \cap \xi^{\perp}\right), \quad \forall \xi \in S^{n-1}
$$

imply

$$
\mu(K) \leq \mu(L) .
$$

Stability in Zvavitch result was established in [26, Theorem 2]. Note that in the case of volume (when $f \equiv 1$ ), the result of Theorem 4.1 is weaker than that of Theorem 2.1. Also, Theorem 4.1 was formulated in [26] for dimensions up to 4 only, however, the proof works in any dimension under the assumption that $K$ is an intersection body.

Theorem 4.1. ([26]) Let $f$ be an even non-negative continuous function on $\mathbb{R}^{n}$, let $\mu$ be the measure with density $f$, let $K$ and $L$ be origin-symmetric star bodies in $\mathbb{R}^{n}$, and let $\varepsilon>0$. Suppose that $K$ is an intersection body and that for every $\xi \in S^{n-1}$,

$$
\mu\left(K \cap \xi^{\perp}\right) \leq \mu\left(L \cap \xi^{\perp}\right)+\varepsilon .
$$

Then

$$
\mu(K) \leq \mu(L)+\frac{n}{n-1} c_{n}|K|^{1 / n} \varepsilon .
$$

Interchanging $K$ and $L$, we get the volume difference inequality.

Corollary 4.2. If $K$ and $L$ are intersection bodies in $\mathbb{R}^{n}$ (in particular, any origin-symmetric convex bodies in $\left.\mathbb{R}^{n}, n \leq 4\right)$, then

$$
\begin{gathered}
|\mu(K)-\mu(L)| \\
\leq \frac{n c_{n}}{n-1} \max _{\xi \in S^{n-1}}\left|\mu\left(K \cap \xi^{\perp}\right)-\mu\left(L \cap \xi^{\perp}\right)\right| \max \left\{|K|^{\frac{1}{n}},|L|^{\frac{1}{n}}\right\} .
\end{gathered}
$$


Sending $L$ to the emply set, we arrive at the hyperplane inequality for arbitrary measure.

Corollary 4.3. If $K$ is an intersection body in $\mathbb{R}^{n}$ (in particular, any origin-symmetric convex body in $\left.\mathbb{R}^{n}, n \leq 4\right)$, then

$$
\mu(K) \leq \frac{n}{n-1} c_{n} \max _{\xi \in S^{n-1}} \mu\left(K \cap \xi^{\perp}\right)|K|^{1 / n} .
$$

The constant in (4.4) is sharp, it is achieved asymptotically when $K=B_{2}^{n}$ and $\mu$ converges weakly to the uniform measure on the sphere $S^{n-1}$; see [26].

Acknowledgements. This work was partially supported by the US National Science Foundation through grant DMS-1001234. I wish to thank the Max Planck Institute for Mathematics for support and hospitality during my stay in Spring 2011, when this project was initiated.

\section{References}

[1] K. Ball. Some remarks on the geometry of convex sets. Geometric aspects of functional analysis (1986/87), Lecture Notes in Math. 1317, Springer-Verlag, Berlin-Heidelberg-New York, 1988, 224-231.

[2] K. Ball. Shadows of convex bodies. Trans. Amer. Math. Soc. 327 (1991), 891-901.

[3] K. Ball. Logarithmically concave functions and sections of convex sets in $R^{n}$. Studia Math. 88 (1988), 69-84.

[4] J. Bourgain. On high-dimensional maximal functions associated to convex bodies. Amer. J. Math. 108 (1986), 14671476.

[5] J. Bourgain. Geometry of Banach spaces and harmonic analysis. Proceedings of the International Congress of Mathematicians (Berkeley, Calif., 1986), Amer. Math. Soc., Providence, RI, 1987, 871-878.

[6] J. Bourgain. On the distribution of polynomials on high-dimensional convex sets. Geometric aspects of functional analysis, Israel seminar (198990), Lecture Notes in Math. 1469 Springer, Berlin, 1991, 127-137.

[7] J. Bourgain. On the Busemann-Petty problem for perturbations of the ball. Geom. Funct. Anal. 1 (1991), 1-13.

[8] H. Busemann, C. M. Petty. Problems on convex bodies. Math. Scand. 4 (1956), 88-94.

[9] R. J. Gardner. Intersection bodies and the Busemann-Petty problem. Trans. Amer. Math. Soc. 342 (1994), 435-445.

[10] R. J. Gardner. A positive answer to the Busemann-Petty problem in three dimensions. Annals of Math. 140 (1994), 435-447.

[11] R. J. Gardner. Geometric tomography. Second edition, Cambridge University Press, Cambridge, 2006.

[12] R. J. Gardner, A. Koldobsky, Th. Schlumprecht. An analytic solution to the Busemann-Petty problem on sections of convex bodies. Annals of Math. 149 (1999), 691-703.

[13] I. M. Gelfand, G. E. Shilov. Generalized functions, vol. 1. Properties and operations. Academic Press, New York, 1964.

[14] I. M. Gelfand, N. Ya. Vilenkin. Generalized functions, vol. 4. Applications of harmonic analysis. Academic Press, New York, 1964.

[15] A. Giannopoulos. A note on a problem of H. Busemann and C. M. Petty concerning sections of symmetric convex bodies. Mathematika 37 (1990), 239-244.

[16] P. Goodey, E. Lutwak, W. Weil. Functional analytic characterization of classes of convex bodies. Math. Z. 222 (1996), 363-381.

[17] E. Grinberg, Gaoyong Zhang. Convolutions, transforms, and convex bodies. Proc. London Math. Soc. (3) 78 (1999), $77-115$.

[18] B. Klartag. On convex perturbations with a bounded isotropic con- stant. Geom. Funct. Anal. (GAFA) 16 (2006), 1274-1290.

[19] A. Koldobsky. An application of the Fourier transform to sections of star bodies. Israel J. Math. 106 (1998), 157-164.

[20] A. Koldobsky. Intersection bodies, positive definite distributions and the Busemann-Petty problem. Amer. J. Math. 120 (1998), 827-840.

[21] A. Koldobsky. Intersection bodies in $\mathbb{R}^{4}$. Adv. Math. 136 (1998), 1-14.

[22] A. Koldobsky. Fourier analysis in convex geometry. Amer. Math. Soc., Providence RI, 2005.

[23] A. Koldobsky. A generalization of the Busemann-Petty problem on sections of convex bodies. Israel J. Math. 110 (1999), 75-91.

[24] A. Koldobsky. Stability in the Busemann-Petty and Shephard problems. Adv. Math. 228 (2011), $2145-2161$.

[25] A. Koldobsky. Stability of volume comparison for complex convex bodies. Arch. Math. (Basel) 97 (2011), 91-98.

[26] A. Koldobsky. A hyperplane inequality for measures of convex bodies in $\mathbb{R}^{n}, n \leq 4$. Dicrete Comput. Geom. 47(2012), 538-547.

[27] A. Koldobsky, Dan Ma. Stability and slicing inequalities for intersection bodies. Geom. Dedicata, 2012, DOI: 10.1007/s10711-012-9729-x

[28] A. Koldobsky, G. Paouris, M. Zymonopoulou. Complex intersection bodies. arXiv:1201.0437.

[29] A. Koldobsky, M. Lifshits. Average volume of sections of star bodies. Geometric aspects of functional analysis, 119-146, Lecture Notes in Math., 1745, Springer, Berlin, 2000. 
[30] A. Koldobsky, D. Ryabogin, A. Zvavitch. Projections of convex bodies and the Fourier transform. Israel J. Math. 139 (2004), 361-380.

[31] A. Koldobsky, V. Yaskin, M. Yaskina. Modified Busemann-Petty problem on sections of convex bodies. Israel J. Math. 154 (2006), 191-207.

[32] A. Koldobsky, V. Yaskin. The interface between convex geometry and harmonic analysis. CBMS Regional Conference Series in Mathematics, 108, American Mathematical Society, Providence, RI, 2008.

[33] D. G. Larman, C. A. Rogers. The existence of a centrally symmetric convex body with central sections that are unexpectedly small. Mathematika 22 (1975), 164-175.

[34] E. Lutwak. Intersection bodies and dual mixed volumes. Adv. Math. 71(1988), 232-261.

[35] V. Milman, A. Pajor. Isotropic position and inertia ellipsoids and zonoids of the unit ball of a normed n-dimensional space. in: Geometric Aspects of Functional Analysis, ed. by J. Lindenstrauss and V. Milman, Lecture Notes in Mathematics 1376, Springer, Heidelberg, 1989, pp. 64-104.

[36] M. Papadimitrakis. On the Busemann-Petty problem about convex, centrally symmetric bodies in $\mathbb{R}^{n}$. Mathematika 39 (1992), 258-266.

[37] C. M. Petty. Projection bodies. Proc. Coll. Convexity (Copenhagen 1965), Kobenhavns Univ. Mat. Inst., $234-241$.

[38] R. Schneider. Zu einem problem von Shephard über die projektionen konvexer Körper, Math. Z. 101 (1967), 71-82.

[39] R. Schneider. Convex bodies: the Brunn-Minkowski theory. Cambridge University Press, Cambridge, 1993.

[40] G. C. Shephard. Shadow systems of convex bodies. Israel J. Math. 2 (1964), 229-306.

[41] V. Yaskin. Modified Shephard's problem on projections of convex bodies. Israel J. Math. 168 (2008), 221-238.

[42] Gaoyong Zhang. Centered bodies and dual mixed volumes. Trans. Amer. Math. Soc. 345 (1994), $777-801$.

[43] Gaoyong Zhang. Intersection bodies and Busemann-Petty inequalities in $\mathbb{R}^{4}$. Annals of Math. 140 (1994), 331-346.

[44] Gaoyong Zhang. A positive answer to the Busemann-Petty problem in four dimensions. Annals of Math. 149 (1999), $535-543$.

[45] Gaoyong Zhang. Sections of convex bodies. Amer. J. Math. 118 (1996), 319-340.

[46] A. Zvavitch. The Busemann-Petty problem for arbitrary measures. Math. Ann. 331 (2005), 867-887. 